\title{
Género y Urbanismo: ¿hacia ciudades más sensibles e inclusivas?
}

\author{
Carmen Innerarity Grau \\ UNIVERSIDAD PÚBLICA DE NAVARRA, PAMPLONA, ESPAÑA \\ carmen.innerarity@unavarra.es \\ Beatriz Acha Ugarte \\ UNIVERSIDAD PÚBLICA DE NAVARRA, PAMPLONA, ESPAÑA \\ beatriz.acha@unavarra.es
}

\author{
Ana Sancho Martínez \\ UNIVERSIDAD PÚBLICA DE NAVARRA, PAMPLONA, ESPAÑA \\ anabm30@hotmail.com
}

\begin{abstract}
Resumen: El objeto de este trabajo es analizar en qué medida los ayuntamientos de las ciudades de Pamplona y Tudela están incluyendo la perspectiva de género en sus políticas de desarrollo urbano. El marco teórico del estudio es, en primer lugar, la crítica feminista a la distinción entre espacio público y privado, -plasmada en un diseño de las ciudades que separa los ámbitos de trabajo de los ámbitos de cuidado- $y$, en segundo lugar, la necesidad de atender a las necesidades específicas de las mujeres -y a su mayor dedicación a las tareas de cuidado- para garantizar un acceso equitativo a la ciudad. Tomando como referencia la documentación emanada del programa Habitat en sus sucesivas reuniones desde la primera conferencia, en 1976, hasta la última, en 2016, elaboramos una serie de recomendaciones que proponemos utilizar como indicadores concretos para evaluar el grado de integración de la perspectiva de género en las políticas de urbanismo. A partir de la sistematización de las propuestas de Habitat, realizamos un análisis de los Planes de Igualdad y de Urbanismo de los municipios seleccionados, complementado con entrevistas al personal técnico en ambas áreas. Los hallazgos muestran un desarrollo desigual de las recomendaciones de Habitat en Pamplona y Tudela, pero, al mismo tiempo, una clara conciencia de que el urbanismo no es neutro en lo que se refiere al género y, concretamente, una revalorización de los cuidados en las políticas, que se traduce en acciones muy concretas para favorecer la corresponsabilidad.
\end{abstract}

Palabras clave: género, urbanismo, igualdad, ciudadanía.

Abstract: The purpose of this work is to analyse the extent to which the local councils of the cities of Pamplona and Tudela are including the gender perspective in their urban development policies. The theoretical framework of the study "is based on", first, the feminist critique of the distinction between the public and the private space, -which leads to a city design where areas for working are separated from areas for caring-and, secondly, the requirement to meet the specific needs of women - including their role as care-givers - to ensure equal access to the city. Based on the documents issued by the Habitat pro- 
gramme in its successive meetings from the first conference, in 1976, until the last one, in 2016, we develop a series of recommendations which may be used as specific indicators to assess the degree of gender mainstreaming in urban planning policies. Based on the systematization of Habitat's proposals, we carry out an analysis of the Equality and Urban Planning Plans of the selected cities, complemented by interviews with members of the technical staff in both areas. Our findings show an uneven development of Habitat's recommendations has taken place in both Pamplona and Tudela; however, a clear awareness also exists that urbanism is not gender-neutral; at the same time, the importance of care provision in urban policies is growing, as can be seen in the introduction of some actions in favour of co-responsibility.

Key words: gender, urbanism, equality, citizenship

\section{Introducción: planteamiento de la investigación ${ }^{1}$}

91 ablar de urbanismo con perspectiva de género parece remitir a temas como la seguridad o la visibilidad de las mujeres, por ejemplo, en los nombres de las calles. Pero, desde la crítica feminista al sesgo de género que encierra la ciudadanía y a la separación entre espacio público y privado, apunta también a la manera en que el urbanismo puede contribuir a remover los obstáculos que impiden a las mujeres acceder en igualdad de condiciones a la esfera pública, y a la ciudad como su plasmación espacial (Innerarity y Sancho, 2015; Sancho, 2020). La demanda feminista por la inclusión en la ciudadanía denuncia que la mera igualdad formal no garantiza la igualdad real. Esto se debe a que el individualismo liberal no toma en consideración aspectos relevantes para las personas que tienen que ver con su identidad, como puede ser el género, y que condicionan su acceso a la esfera pública. Es más, "toda abstracción de género neutro termina siendo sospechosamente masculina" (Phillips, 2002: 2), de forma que el "individuo" resulta ser un varón. Por ello, autoras como Mouffe (2001), Pateman (1988, 1995), Okin (1989, 1994, 2004), Mansbridge y Okin $(2007)$ o Young $(1990,1996)$ proponen elaborar una concepción sexualmente diferenciada de la individualidad que atienda a la manera específica en que varones y mujeres se incorporan a la ciudadanía y, por lo tanto, acceden a la ciudad.

En este sentido, nuestro estudio parte de la tesis de que las ciudades han sido diseñadas conforme a la división de espacios público y privado y a la atribución de uno y otro a varones y mujeres respectivamente, considerando que unas personas trabajan y otras cuidan, que en unos lugares se trabaja y en otros se cuida. Existe, ciertamente, una igualdad formal en el acceso a la ciudad; sin embargo, la división de espacios impide la igualdad real de quienes soportan en mayor medida las tareas

1. El proyecto que ha permitido obtener estos resultados ha recibido financiación de la Fundación "la Caixa" (ID 100010434), acuerdo LCF/PR/PR15/51100007. 
de cuidado -las mujeres- (Sánchez de Madariaga, 2009; Durán, 2010 y 2017), aunque solo sea por el tiempo invertido en transitar del espacio de los cuidados al espacio del trabajo y viceversa.

Todo ello aplicado al urbanismo significa dos cosas. En primer lugar, frente al universalismo abstracto, la necesidad de incluir la experiencia y condiciones específicas de las mujeres en las decisiones que tienen que ver con el diseño de las ciudades (Muxi et al., 2011; Falú, 2013). Y, en segundo lugar, frente a la división de espacios, es preciso "poner en igualdad de condiciones las exigencias derivadas del mundo productivo y las derivadas del mundo reproductivo, es decir, las necesidades cotidianas de atención a las personas" (Muxí et al., 2011: 108). Sin dejar de atender a los aspectos más tradicionales del urbanismo con perspectiva de género, como la seguridad o la visibilidad, queremos añadir a nuestro análisis esta doble perspectiva, que atiende a las condiciones específicas de las mujeres y a la unión entre espacio público y espacios de cuidado, en definitiva, a la igualdad en el acceso a la ciudad.

Desde este marco teórico, el objeto de esta investigación es analizar en qué medida los ayuntamientos de las ciudades de Pamplona y Tudela van incorporando la perspectiva de género en sus políticas con el fin de diseñar ciudades realmente inclusivas. Esta investigación entronca con los hallazgos obtenidos previamente respecto a otras ciudades -Bilbao, Zaragoza, Barcelona, entre otras (Innerarity y Sancho, 2015)- y nos permite avanzar en el conocimiento de las diferentes formas en las que los entes municipales en España han abordado la cuestión del vínculo entre el género y las políticas urbanísticas.

\section{Metodología}

Para cumplir nuestro objetivo hemos llevado a cabo un análisis de los Planes de Igualdad y de Urbanismo -centrándonos en los actualmente vigentes- de los municipios de Pamplona y Tudela. Ciertamente, los Planes son solo uno de los múltiples instrumentos que un ente local puede utilizar para materializar sus preferencias en relación con las políticas públicas, por lo que nuestra visión es exploratoria un análisis exhaustivo del compromiso con los temas de género exigiría considerar también otro tipo de medidas desarrolladas por los ayuntamientos. Por ello, y con el fin de contrastar los resultados derivados del análisis documental, este se ha complementado con entrevistas al personal técnico de ambas áreas, entre noviembre de 2018 y marzo de 2019. Adicionalmente, hemos recogido información sobre otro tipo de actuaciones municipales en este campo.

Se han seleccionado estos dos municipios por ser los de mayor tamaño poblacional de Navarra. Con casi 200.000 habitantes censados en Pamplona y apenas 35.000 
en Tudela ${ }^{2}$, partimos de la hipótesis de que la actividad desplegada por el Consistorio pamplonés en el campo de nuestro interés (políticas de urbanismo y de igualdad) probablemente será muy superior, cuantitativa y cualitativamente hablando, a la del ayuntamiento tudelano. Sin embargo, esta variable por sí sola podría no explicar las diferencias entre ambos municipios, de haberlas. En este sentido, nuestro estudio no pretende ser meramente descriptivo, sino que aspiramos a entender y a explicar por qué hay distintas maneras de incorporar los temas de igualdad al urbanismo en la agenda local.

En último término, pretendemos averiguar si los ayuntamientos recogen, no sólo aquellos aspectos que, como la seguridad o la visibilidad, son los tradicionales del urbanismo con perspectiva de género -a los que, por tanto, es probable (y esta es una segunda hipótesis de investigación) que hayan prestado una mayor atención-; sino, sobre todo, comprobar en qué medida se van incluyendo también otro tipo de cuestiones -como las que tienen que ver con el cuidado-, que están en la raíz de la desigualdad en el acceso a la ciudad. Esto nos permitirá valorar el compromiso real -al menos, a nivel conceptual y propositivo- con un urbanismo con perspectiva de género.

En Innerarity y Sancho (2015) definimos una serie de indicadores para analizar la inclusión de la perspectiva de género en la planificación de las ciudades a partir de las recomendaciones del Programa Habitat I y II de Naciones Unidas, de 1976 y 1996, respectivamente, y de la Carta Europea de la Mujer en la Ciudad, de 1995. Esa batería de indicadores (ver cuadro 1) es la que ha orientado tanto el análisis documental como las entrevistas realizadas. La aportación de este estudio radica, así, no sólo en el análisis de nuevos casos -que permitirá construir progresivamente un mapa de la inclusión de la perspectiva de género en las políticas municipales- sino, sobre todo, en el hecho de que a las propuestas de entonces añadimos ahora el contenido de la última Conferencia Habitat III, celebrada en 2016.

\section{La ciudad con perspectiva de género: la Nueva Agenda Urbana de Habitat III}

La cuestión del urbanismo con perspectiva de género ha resultado primordial para la Organización Nacional de las Naciones Unidas en el marco de las Conferencias Habitat I (1976), Habitat II (1996) y la posterior Declaración de Estambul sobre los asentamientos humanos (1996) y Habitat III (2016). Concretamente, la Declaración de Estambul (ONU 1996a) supuso una llamada de atención sobre la necesidad de lograr que los gobiernos integren la perspectiva de género en las políticas relativas a los asentamientos humanos. Desde entonces, ha sido constante el

2. Según datos de Padrón de 1 de enero de 2019, Pamplona tiene una población de 199.066 habitantes y Tudela de 35.593 (información disponible en el Instituto de Estadística de Navarra: https://administracionelectronica. navarra.es/GN.InstitutoEstadistica.Web/informacionestadistica.aspx?R=1\&E=1). 
compromiso de Naciones Unidas con una política de planificación urbana sensible al género (Sancho, 2016).

\section{Cuadro 1}

Recomendaciones sobre urbanismo y género (Programa Habitat)

1. Gobernanza

2. Participación de las mujeres en el diseño de la ciudad

3. Igualdad en el acceso a la vivienda

4. Incrementar la formación en igualdad

5. Visibilizar a las mujeres

6. Implicación de medios de comunicación

7. Necesidad de seguridad para el uso del espacio público

8. Acceso a la ciudad

Fuente: elaboración propia

En 2016 la Conferencia Habitat III culminó con la aprobación de la Nueva Agenda Urbana (en adelante, NAU), documento que "ha establecido un marco normativo que ha guiado las políticas hasta llegar a la actualidad” (Acero et. al., 2019: 24) y que supone, probablemente, una oportunidad definitiva para afrontar los retos que plantea la sostenibilidad de las ciudades, también por lo que respecta al género. Las recomendaciones de Habitat y, concretamente, la NAU, constituyen un referente que ha orientado el trabajo posterior -en los ámbitos académico y asociativo, fundamentalmente- que busca traducir el modelo de ciudad propuesto en medidas concretas de acción para incluir la visión de género en las políticas urbanas (Boccia, 2020).

La NAU define los rasgos que caracterizan a la "ciudad ideal" y que exigen superar un enfoque universalista, "ciego a las diferencias", para situar a la persona y sus circunstancias específicas -entre ellas, el género- como foco que oriente el diseño de las políticas públicas. Este modelo se traduce en una serie de compromisos y medidas concretas de acción:

En primer lugar, es preciso promocionar una planificación sensible que incluya a las mujeres y sus específicos usos del espacio y facilite su acceso en igualdad de 
condiciones (ONU, 2017: 35), invirtiendo en sistemas de movilidad urbana, desarrollando un diseño de equipamientos y servicios sensibles al género (ONU, 2017: 114). Esta recomendación, que hemos incluido bajo el epígrafe de gobernanza, se concreta en acciones como establecer canales de comunicación entre las áreas encargadas de Urbanismo e Igualdad, introducir elementos de análisis y medida del impacto de igualdad de las acciones con indicadores desagregados por género o elaborar presupuestos sensibles al género (ONU, 2017: 92, 151).

En segundo lugar, la NAU destaca la importancia de empoderar a las mujeres para la participación en la toma de decisiones (ONU, 2017: 13). Esto se traduce en impulsar canales establecidos de participación/recogida de los intereses de las mujeres o en contar con el movimiento asociativo para la elaboración de planes de acción (Carta, 1995: 15; ONU, 1996a: 15; ONU, 2017: 48, 92).

La tercera recomendación se refiere a la vivienda. Dada la insuficiencia de las políticas dirigidas a toda la ciudadanía, es preciso elaborar medidas especiales que garanticen la igualdad para "acceder, poseer y controlar el suelo y la vivienda" (ONU, 1996a: 3; ONU, 2017: 14).

En cuarto lugar, se insiste en la importancia de la formación en igualdad de los agentes implicados en las políticas (Carta, 1995, principios 9 y 10), la visibilidad simbólica de las mujeres (Carta, 1995: 2; ONU, 2017: punto 155), -nombres de calles o edificios- y la actuación sobre los medios de comunicación y la opinión pública para combatir los estereotipos de género (Carta, 1995:11).

Otra de las recomendaciones apunta a "promover un entorno seguro, saludable e inclusivo en las ciudades y los asentamientos humanos (...), teniendo en cuenta que las mujeres y las niñas, los niños y los jóvenes y las personas en situaciones vulnerables suelen verse especialmente afectados" (ONU, 2017: 39). Para ello es necesaria la "instauración de redes bien diseñadas de calles y otros espacios públicos seguros, ecológicos y de calidad que sean accesibles para todos y estén libres de delincuencia y violencia, en particular libres de acoso sexual y violencia por razón de género" (ONU, 2017: 100).

En esta línea, Habitat recomienda, por último, actuar sobre las condiciones de acceso a la ciudad. El acceso se entiende tanto en sentido físico -transporte (Carta, 1995: 7; ONU, 1996b: 4; ONU, 2017: 13)- como, sobre todo, en sentido simbólico. Concretamente, dado que muchas de las desigualdades hunden sus raíces en las condiciones de la vida privada y, de manera específica, en el hecho de que las tareas del cuidado aún recaen en mayor medida sobre las mujeres, es preciso facilitar tanto la movilidad, que permita superar la división de espacios, como el acceso a los diferentes servicios del municipio (ONU, 2017: 95), especialmente a los servicios que ayudan al cuidado (Carta, 1995: 8; ONU, 2017: 32). 
Estas recomendaciones de Habitat sobre urbanismo y género pueden resumirse en una serie de medidas que proponemos utilizar como indicadores concretos para evaluar en qué medida los planes de las ciudades de Pamplona y Tudela integran la perspectiva de género. A partir de todos ellos procedemos a analizar la documentación escrita y las entrevistas realizadas en cada uno de los dos municipios navarros. El objetivo no es tanto realizar una comparación sistemática de las similitudes y diferencias entre las dos ciudades, sino reflexionar sobre las maneras en que los municipios diseñan y elaboran sus políticas en este campo, para, a futuro, poder contrastar hipótesis sobre las razones por las que existen diferentes planteamientos en esta cuestión.

\section{Análisis: El género en los planes de Pamplona y Tudela}

A continuación, pasamos a exponer los resultados del análisis de la documentación y de las entrevistas al personal técnico municipal de ambos ayuntamientos ${ }^{3}$.

En primer lugar, se hicieron una serie de preguntas de carácter general sobre el conocimiento de Habitat y sobre la inclusión de la perspectiva de género en su área de trabajo. Todas las personas entrevistadas señalaron tener un conocimiento escaso del programa Habitat.

En cuanto a la valoración de la inclusión de la perspectiva de género en las políticas y la colaboración entre las dos áreas, el personal de Pamplona señala que no hay proyectos conjuntos, aunque sí contactos puntuales en cuestiones concretas, como la redacción de la nueva Ordenanza de Igualdad (2019) ${ }^{4}$. Por otro lado, desde el área de Urbanismo de Pamplona señalan que las primeras preocupaciones por el tema del género se dieron en la redacción del Plan Municipal (2002): en ese momento se pidió colaboración tanto al área de Igualdad, como a un grupo de mujeres urbanistas, cuyas indicaciones fueron recogidas en el Plan. Además, se mantienen reuniones con Igualdad, en las que se explican los proyectos que están desarrollando, aunque no han proporcionado un catálogo de acciones concretas (TUP2).

En cualquier caso, el III Plan de Igualdad de Pamplona $(2016$ - 2022)5 (en adelante IIIPIP) plantea como primer objetivo "Garantizar desde el Ayuntamiento de Pamplona un compromiso real con la igualdad de las mujeres haciendo del principio de igualdad un principio de gobernabilidad" (IIIPIP, 28). Esto exige, entre otras cosas, la colaboración entre las diferentes áreas municipales. Para ello propone establecer mecanismos organizativos de coordinación y, concretamente, la creación de

3. A efectos de clarificación, hemos denominado al Personal Técnico de Igualdad de Pamplona TIP; al Personal Técnico de Urbanismo de Pamplona TUP1 y TUP2; al Personal Técnico de Igualdad de Tudela, TIT; y al Personal Técnico de Urbanismo de Tudela, TUT.

4. Aprobada el 7 de marzo de 2019.

5. Aprobado el 7 de abril de 2016. 
una Unidad de Igualdad, que dé "apoyo técnico para la transversalidad de género en cada área municipal" (IIIPIP, 30). Además, el plan incluye medidas que afectan a ambos departamentos y así lo dice expresamente al indicar los correspondientes responsables o al establecer con carácter general el objetivo del "incremento de las áreas implicadas en la aplicación práctica del principio de igualdad y en el desarrollo del plan" (IIIPIP, 28).

En Tudela no existía, al comienzo de nuestra investigación, ninguna relación entre las dos áreas municipales, ni la ha habido históricamente, a pesar del interés en la materia mostrado por el personal del área de Igualdad (TIT). En el pasado sí se había desarrollado algún proyecto común entre las dos áreas, como el curso "Urbanismo con perspectiva de género" ${ }^{\text {, }}$ que fue bien valorado por la Concejala y Directora de entonces, pero no consiguió el apoyo del arquitecto y del arquitecto técnico. Más aún, nada de lo propuesto se llegó a llevar a cabo, porque se produjo un cambio de gobierno. En general, las áreas de Urbanismo e Igualdad de Tudela parecen haber trabajado en aparente desconexión hasta hace muy poco tiempo.

Una vez realizada la valoración general, pasamos a exponer los resultados del cumplimiento de los indicadores elaborados a partir de la documentación del programa Habitat.

\subsection{Gobernanza}

A pesar de que en Pamplona no existen, con carácter general, datos desagregados ni presupuestos desglosados, sí se observa una voluntad de incluir la mirada de género. De hecho, la persona entrevistada del área de Igualdad se refiere a "otra manera de hacer política" (TIP), que significa que "en todos los temas que afectan al Ayuntamiento se intenta aplicar la perspectiva de género" (TIP). Así lo recogen tanto la Ordenanza como el Plan recientemente aprobados. Hay una conciencia clara de que "El urbanismo no es neutro desde el punto de vista del género...puede mejorar o, por el contrario, puede dificultar la manera en que las mujeres llevan a cabo sus múltiples actividades, tanto en la esfera pública como privada" (TIP).

En esta línea, "se va a trabajar en los informes de impacto de género" (TIP). Y, de hecho, la ordenanza municipal (OI, 2019: art. 25-29, art. 30-32) es -a juicio del personal de Igualdad- más ambiciosa que la propia Ley de Contratos del Sector Público 7 ,

6. Organizado por el Área de Asuntos Sociales y Mujer del Ayuntamiento en mayo de 2010, contó con una nutrida asistencia del Área de Urbanismo, Vivienda y Obras.

7. Ley 9/2017, de 8 de noviembre, de Contratos del Sector Público, por la que se transponen al ordenamiento jurídico español las Directivas del Parlamento Europeo y del Consejo 2014/23/UE y 2014/24/UE, de 26 de febrero de 2014 (disponible en: .https://www.boe.es/buscar/pdf/2017/BOE-A-2017-12902-consolidado.pdf). 
que solo pide estos informes en los superiores a 5 millones de euros. En el Plan de Igualdad se detalla "la incorporación de la perspectiva de género en todos los planes, programas y proyectos del Ayuntamiento de especial relevancia y adjuntar para su aprobación un informe de impacto de género" (IIIPIP, 32). El objetivo es, en definitiva, "que la igualdad competa a todas las áreas y que todas las áreas trabajen desde un enfoque de género", siendo conscientes de que "esas políticas que se están desarrollando tienen un impacto diferente para hombres y mujeres... Tendrán que analizar si esas políticas favorecen la igualdad o están perpetuando un sistema desigual" (TIP; OI, 2019: título 2 y capítulo 3).

Desde Urbanismo (TU2P), en el Ayuntamiento de Pamplona se considera que las cuestiones relativas al género no se plantean siempre de manera explícita, ya que sus acciones se refieren al espacio público en general, a toda la ciudadanía, pero eso no quiere decir que el tema no esté presente. De hecho, en acciones específicas sobre la ciudad construida sí se incluyen medidas concretas orientadas a la igualdad, que "tienen que ver con la calidad del espacio público, con su claridad, con su alumbrado en horas nocturnas, con su conformación física, con la transparencia y la visión, con la accesibilidad" (TU2P). Deben rellenar fichas sobre el impacto de género, dentro de "las acciones concretas que llevamos a cabo para la igualdad" (TU2P), como accesos transparentes a los nuevos ascensores o evitar puntos oscuros. Pero "la perspectiva de género está integrada en la actuación general" (TU2P). La prioridad es que el espacio público sea inclusivo.

Por lo que respecta al municipio de Tudela, la relación entre las dos áreas, como ya se ha mencionado, ha sido muy escasa hasta la fecha, lo que se ha traducido en la ausencia de contenidos de trabajo compartidos y en la práctica inexistencia de relaciones interdepartamentales. Así, la perspectiva de género no está incluida en el aún vigente PGOU del municipio tudelano, aprobado en 1991 y refundido en 1996; como tampoco se ha recogido directamente en el EMOT (documento de Estrategia y Modelo de Ocupación del Territorio ${ }^{8}$ ), que sienta las bases para el posterior desarrollo del planeamiento urbanístico del municipio y es previo a la elaboración de un nuevo PGOU. En el momento en el que se realizaron las entrevistas para esta investigación, en el área de Urbanismo sí había constancia del interés en el tema por parte de la Dirección de quien dependía Igualdad, y se consideraba que el momento era idóneo para incorporar aportaciones de la ciudadanía o, específicamente, de las mujeres.

8. EI EMOT se ha redactado como primer documento del Plan General Municipal en cumplimiento del artículo 58.2 del DFL 1/2017, por el que se aprueba el Texto Refundido de la Ley Foral de Ordenación del Territorio y Urbanismo (en http://www.tudela.es/docs/urbanismo/emot/2019/memoria-emot-tudela-ene-2019.pdf). 
Por su parte, el vigente III Plan de Igualdad de Mujeres y Hombres del Ayuntamiento $2018-2021^{9}$ (en adelante IIIPIT) sí menciona de manera específica al área de Urbanismo, al desplegar el segundo eje de intervención, denominado "Territorio seguro y libre de violencia contra las mujeres". En él se define, de manera concreta, la estrategia de "eliminar los lugares y espacios de mayor inseguridad y riesgo para las mujeres", y se menciona a Urbanismo, entre los agentes implicados en esta actuación (IIIPIT: 25).

En cuanto a la existencia de indicadores desagregados por género, para el personal técnico del área de Urbanismo, no existen, si bien "los criterios sociales sí aparecen en los pliegos: que haya mujeres en la participación del equipo" (TUT). Sin embargo, el IIIPIT sí alude a la desagregación de datos por sexo. Así se recoge en un primer eje de actuación, el de Gobernanza local y Transversalidad de género: "Se trata de crear estructuras que garanticen el desarrollo de las políticas de igualdad, así como procedimientos de trabajo para la transversalidad de género y de capacitar al personal de la administración local". Por ello, con el objetivo de que todos los procedimientos de la administración local la incorporen, se propone incluir la perspectiva de género en cláusulas de subvenciones, convenios y contrataciones, así como en la elaboración del presupuesto municipal y en la recogida de datos, información y estadísticas de municipio (p. 23).

\subsection{Participación}

Dentro de la línea estratégica "Participación e incidencia política" del IIIPIP, el consistorio pamplonés propone "conseguir que el movimiento organizado de mujeres se convierta en un órgano de interlocución del Ayuntamiento" (IIIPIP: 42). Como medidas concretas señala revisar los mecanismos estables de participación para incluir la igualdad entre hombres y mujeres, fomentar la participación de las asociaciones y colectivos en el diseño e implementación de las políticas municipales de igualdad e incluir incluir sus reivindicaciones en las planificaciones festivas, así como crear de un espacio, tanto físico como virtual, de encuentro entre las mujeres de la ciudad. De hecho, ya la Ordenanza de Igualdad recientemente aprobada incluye las aportaciones de colectivos que colaboraron en su elaboración (TIP).

En Tudela, el IIIPIT define un amplio eje de actuación (4) titulado "Empoderamiento de las mujeres, participación sociopolítica y cambio de valores", que tiene como objetivo la participación de mujeres en los procesos de influencia y toma de decisiones, garantizando su participación en igualdad en todos los espacios sociopo- 
líticos y económicos", y que contempla la necesidad de "Incorporar en los procesos de participación criterios que velen por la igualdad de mujeres y hombres en los mismos" (p. 33). Más aún, en otro ámbito y con el objetivo de convertir a Tudela en "territorio seguro" para las mujeres (Eje 2), se estima que se deben "Identificar los Puntos Críticos y sistematizarlos en un Mapa de "Puntos de Riesgo", favoreciendo, especialmente, la participación de las mujeres” (p. 25).

\subsection{Vivienda}

En Pamplona no se han desarrollado medidas específicas en vivienda. Tampoco existen datos de beneficiarias de programas en este ámbito, aunque "es una demanda desde el área de Igualdad que todos los datos estén desagregados por sexo" (TIP).

Tampoco en Tudela se ha incorporado la vivienda al IIIPIT, ni desde Urbanismo se han pensado políticas específicamente destinadas a las mujeres, si bien el personal técnico era claramente favorable a su valoración (por ejemplo, en lo relativo a viviendas destinadas a las mujeres víctimas de violencia de género) en el futuro PGOU.

\subsection{Formación en igualdad}

El personal de Urbanismo de Pamplona señala que no hay planes específicos para la formación en igualdad, pero sí acciones concretas desde la sensibilidad personal y, desde luego, lo consideran interesante, tanto a nivel personal como para el Ayuntamiento. "A la hora de construir un edificio, qué parámetros hay que tener en cuenta, igual que ocurre con los temas de accesibilidad" (TUP1). Esta necesidad de formación fue subrayada en el proceso de elaboración del Plan, que, de hecho, recoge expresamente el objetivo de "generar conocimiento y competencia suficiente entre el personal político y técnico de todos los niveles para integrar la igualdad en la gestión municipal" (IIIPIP: 31).

Por otra parte, en las convocatorias del área de Urbanismo "no la pides específicamente, pero la habríamos valorado" (TUP1) si hubiese habido alguien que hubiera presentado como mérito la formación en igualdad. Lo que sí se ha recogido en alguna convocatoria es la inclusión de mujeres en el equipo de trabajo. Por ejemplo, para el mantenimiento de jardines, tuvieron en cuenta el tema de la paridad y en las cláusulas sociales se incluye: "De las nuevas contrataciones, el 50\% tenían que ser mujeres y se tenía que empezar por una mujer y tenía que ser alternativo” (TUP1).

El III Plan de Igualdad de Tudela sí recoge la necesidad de elaborar planes formativos dirigidos al personal técnico y político municipal (p. 21). Se trata de conseguir la capacitación técnica y política para la incorporación del enfoque integrado de 
género en el municipio, y de que "el personal político y técnico de la administración local incorpore la mirada de género en sus prácticas” (p. 22).

\subsection{Visibilidad}

Como hemos señalado, este es un aspecto tradicional del urbanismo con perspectiva de género $y$, en consecuencia, algo a lo que ambos consistorios conceden significativa importancia. En concreto, en Pamplona, el IIIPIP pretende "compensar la violencia simbólica ejercida contra las mujeres haciéndolas visibles y reconociendo sus aportaciones al diseño de la ciudad" (IIIPIP: 72). Para ello se propone promover la presencia de mujeres en la denominación de calles, edificios o salas de uso público, "especialmente en los barrios nuevos" (TUP2).

Igualmente, en Tudela, en calles y plazas (nuevas) se tiene en cuenta los nombres de mujeres desde el IIPIT; se han acometido varias iniciativas en este sentido, como el "Estudio sobre la situación de las mujeres de Tudela" de 2004, u otro sobre "La Historia de las mujeres de Tudela durante los últimos 60 años", llevado a cabo en 2010. Asimismo, el Ayuntamiento trabaja desde hace varios años desde la Escuela de Empoderamiento, donde se da importancia a la visibilización y a la participación de las mujeres. Por otra parte, y pese a desconocer las recomendaciones de Habitat en este sentido, el personal de Urbanismo estima también que se podrían hacer ciertas cosas como en otros barrios/municipios, donde hay "paseos Jane Jacobs" (TUT), por ejemplo.

\subsection{Medios de comunicación}

En Pamplona sucede en este ámbito algo semejante a lo que hemos visto en otras cuestiones: se destaca en notas de prensa la imagen que el Ayuntamiento quiere potenciar, "pero sin hacer hincapié de forma más o menos sistemática en los temas de género" (TUP2). En este sentido, el IIIPIP abre una oportunidad, al establecer como línea estratégica: "Facilitar la implicación de los medios de comunicación como informantes de calidad y generadores de opinión y cuestionamiento de los valores tradicionales asociados a la desigualdad" (IIIPIP: 50). Esta es una vía de indudable utilidad para facilitar un cambio de valores que puede permitir avanzar en la igualdad de género de una manera más profunda que otro tipo de cuestiones que, como la mera visibilidad de las mujeres en los nombres de las calles, siendo importantes, no van a la raíz de las situaciones que generan desigualdad y exclusión social.

En Tudela el III Plan de Igualdad no recoge menciones específicas a los medios de comunicación, pero incide en la necesidad de sensibilizar a la ciudadanía sobre "la igualdad como valor social y actuar (...) sobre los estigmas que recaen sobre las mujeres” (p.34). 


\subsection{Seguridad}

Como cabía esperar, este es un campo que recibe especial atención. "Una vida libre de violencia contra las mujeres en una ciudad segura" es una de las líneas estratégicas del IIIPIP. Medidas concretas para el "incremento en la percepción de seguridad por parte de las mujeres de Pamplona” (IIIPIP: 54) son: continuar el trabajo de identificación y eliminación de puntos inseguros, especialmente durante las fiestas locales, realizar un seguimiento activo de la evolución de la seguridad en los barrios de la ciudad diseñando mecanismos estables de coordinación entre las mujeres y el área de urbanismo, sistematizar criterios para evitar la creación de nuevos puntos críticos, disponibilidad de información en materia de violencia de género en el municipio.

Desde el Área de Igualdad de Pamplona (TIP), los temas relativos a la seguridad se trabajan en aspectos concretos, como la campaña contra agresiones durante las fiestas, en coordinación con colectivos de los barrios que organizan diferentes actos. Se aprovecha esa coordinación para recoger cuáles son los puntos críticos del barrio, y no solo durante las fiestas. Algunas de las medidas propuestas son: trabajar sobre los barrios nuevos, con muchos solares y escasa iluminación, o fomentar que las bajeras sean ocupadas por comercio de proximidad. Además, hay una línea de trabajo abierta con la Mancomunidad de la Comarca de Pamplona (responsable de la gestión del transporte urbano) para aumentar frecuencias o revisar la ubicación de las paradas nocturnas.

La cuestión de la seguridad es uno de los ámbitos principales de actuación del área de Urbanismo de Pamplona para una ciudad inclusiva (TUP2), donde tratan los temas que tienen que ver con la iluminación a partir de los datos que proporciona el área de Igualdad (TUP1). Habitualmente funcionan con criterios de eficiencia y bajo consumo, pero si hay una zona que necesita especial iluminación -como los recovecos creados por los ascensores que sobresalen de las fachadas- se le da un tratamiento diferente. Igualmente, los cierres de los comercios deben estar en la línea de fachada, pero todo ello se hace por seguridad en general, sin tener en cuenta de forma específica el género.

¿Y qué ocurre en Tudela? El IIIPIT define cuatro grandes ejes de intervención, entre ellos el de convertir Tudela en "Territorio seguro y libre de violencia para las mujeres", y plantea como estrategia eliminar los lugares y espacios de mayor inseguridad y riesgo para las mujeres, así como identificar "un mapa de Puntos de Riesgo" (p. 25, Eje 2). Se trata de que Tudela sea un "entorno seguro para las mujeres, en el que se tenga conocimiento y conciencia sobre la violencia contra las mujeres, como expresión extrema de la desigualdad y sus diversas formas, así como la actuación coordinada de los servicios públicos necesarios". Y se aspira también a capacitar al personal municipal en esta materia y a promover "la concienciación ciudadana y el 
cambio de valores, a través de la eliminación de estereotipos y roles de género, así como la prevención y erradicación de la violencia contra las mujeres" (ibíd.: 26-27). Es ésta, pues, una cuestión muy importante para Igualdad. Y también se apunta a ella desde Urbanismo, pues se menciona, como un ejemplo a seguir (por su concienciación e implicación en otros contextos urbanos), al "colectivo URBANAS, que se interesaba por si había puntos críticos...que es lo que en el fondo se hace en este trabajo, identificar puntos dudosos" (TUT).

\subsection{Acceso a la ciudad}

Como hemos señalado, aquí se incluyen tanto las medidas relativas al transporte como -sobre todo- las que tienen que ver con los cuidados.

En este ámbito de actuación, en Pamplona ocurre lo mismo que veíamos en relación con la seguridad. La planificación urbanística, la accesibilidad, la dotación de servicios, se hace desde un enfoque integral, sin pensar expresamente en el género (TUP2), buscando que el espacio público sea inclusivo con carácter general.

En cuanto a la accesibilidad y la separación de espacio público y privado, el IIIPIP incluye tres líneas estratégicas vinculadas con la planificación urbana y la cuestión de los cuidados: "Revalorización de los cuidados e interdependencia del sistema productivo y reproductivo", "Desmontar la vinculación mujer-cuidado" y, sobre todo, "Repensar la ciudad desde el modelo de la sostenibilidad de la vida". La permanencia de la asignación tradicional de las tareas de cuidado a las mujeres "dificulta enormemente el desarrollo del derecho efectivo de la ciudadanía de las mujeres" (IIIPIP: 53). Es, por tanto, necesario construir "nuevos modelos de relación entre mujeres y hombres, nuevos usos de los espacios y de los tiempos" (IIIPIP: 53), "repensar la ciudad desde estos nuevos parámetros" (IIIPIP: 53). Se trata de "situar los cuidados en el centro de la vida" con la consiguiente "necesaria articulación de recursos para su soporte" (IIIPIP: 54). Concretamente, propone "mejorar el acceso y condiciones de los servicios de cuidado" (IIIPIP: 57), incluyendo acciones relativas a las personas cuidadoras en el domicilio, a la promoción de iniciativas como los Bancos de Tiempo, la "elaboración y difusión de un mapa de los recursos para el cuidado existentes en la ciudad" o la creación de "recursos para cubrir las necesidades de cuidado" (IIIPIP: 58).

Por su parte, en Tudela ya en 2015 y desde el anterior Plan de Igualdad, se abordó la cuestión del acceso a la ciudad por parte de las mujeres ${ }^{10}$. El III y vigente Plan de

10. En concreto, se llevó a cabo una encuesta sobre "Transporte Público Urbano de Tudela" impulsada por el II Pacto por la Conciliación de la Vida Familiar, Laboral y Personal de Tudela, foro integrado por 22 organizaciones y asociaciones de Tudela y coordinadas por el Ayuntamiento. La acción se enmarcaba en el Eje Conciliación-Corresponsabilidad del II Plan de Igualdad de Oportunidades entre Mujeres y Hombres del Ayuntamiento de Tudela. 
Igualdad no incluye referencias específicas al tema del transporte, pero sí alude de manera muy concreta a la otra manera de entender este acceso a la ciudad en clave de separación de espacios público/privado. Así, hay todo un nuevo eje de intervención que se destina a la conceptualización de un nuevo "Contrato Social" centrado en la corresponsabilidad, la conciliación y los cuidados. Se pretende desde aquí aumentar la "calidad de vida de la ciudadanía, a través del desarrollo de la ética del cuidado y la conciliación corresponsable de la vida laboral familiar y personal de mujeres y hombres" con el objetivo de "visibilizar la importancia de los cuidados para mantener las estructuras sociales y dignificar las condiciones de quienes los asumen". Este esfuerzo pasa también por introducir nuevos "modelos de gestión del tiempo, de los espacios y (una) nueva ética de los cuidados" (IIIPIT, 28-29). La sintonía con el planteamiento de Habitat es, en este punto, altísima.

\section{Cuadro 2.}

La inclusión de las recomendaciones sobre urbanismo y género en los municipios de Pamplona y Tudela

\begin{tabular}{|l|c|c|}
\hline & Pamplona & Tudela \\
\hline Gobernanza & $\mathrm{x}$ & $\mathrm{x}$ \\
\hline Participación de las mujeres en el diseño de la ciudad & & $\mathrm{x}$ \\
\hline Igualdad en el acceso a la vivienda & & $\mathrm{x}$ \\
\hline Incrementar la formación en igualdad & & $\mathrm{x}$ \\
\hline Visibilizar a las mujeres & $\mathrm{x}$ & $\mathrm{x}$ \\
\hline Implicación de medios de comunicación & $\mathrm{x}$ & $\mathrm{x}$ \\
\hline Seguridad para el uso del espacio público & & \\
\hline Acceso a la ciudad & & \\
\hline
\end{tabular}

Fuente: elaboración propia

\section{Resultados}

Del análisis de la documentación y de las entrevistas en Pamplona y Tudela se deduce que el conocimiento de las recomendaciones de Habitat es escaso y que hasta la fecha parece haber habido pocos canales estables de comunicación o proyectos conjuntos entre las áreas de Urbanismo e Igualdad, sobre todo en Tudela. En Pamplona, los planes recientemente aprobados sí recogen la necesidad de institucionalizar de 
alguna manera la colaboración inter-áreas, así como medidas de acción que afectan a ambas. Por contra, y en cuanto a los indicadores desarrollados a partir de Habitat, el eje de Gobernanza en Pamplona es uno de los menos desarrollados en lo relativo al desglose de datos y a los indicadores del impacto de las políticas en relación con el género, mientras que el IIIPIT sí recoge de manera específica esta medida. En ninguno de los dos casos hay datos desagregados sobre el acceso a la vivienda (o medidas para facilitarlo): las estrategias recogidas en los planes se centran sobre todo en medidas urbanísticas referidas al espacio público en general y, por lo tanto, se dirigen a toda la ciudadanía. Tampoco hay acciones concretas en relación con los medios de comunicación en ninguno de los dos casos. Sí que se contemplan, en cambio, en ambos planes, medidas específicas de formación en igualdad, necesidad también señalada por las personas entrevistadas. Respecto a la participación, aunque no existan mecanismos formales, sí se ha recurrido de manera informal a diferentes colectivos para la elaboración de planes y acciones.

El seguimiento de las recomendaciones de Habitat es también parcial en otros indicadores: seguridad y acceso (al espacio físico). En cuanto a la seguridad, en ambos municipios están contempladas acciones muy concretas en este campo. En ocasiones se trata de medidas diseñadas tomando en consideración el género de forma explícita, pero en la mayoría de los casos se derivan de la importancia de crear espacios seguros para toda la ciudadanía. Igualmente, el tema del acceso se trata, con carácter general, desde el objetivo de diseñar ciudades inclusivas, también en relación con otros colectivos como el de personas discapacitadas, pero sin referencias explícitas a las mujeres del interés municipal por este tema.

Por contra, la cuestión de la visibilidad, tema clásico del urbanismo con perspectiva de género, es tangible en las políticas de los dos Ayuntamientos, especialmente en los barrios más modernos de ambas ciudades, que han procurado incluir nombres de personajes femeninos. Como también lo es la del acceso de las mujeres al espacio público en general en igualdad de condiciones: cabe destacar la importancia de la necesidad de repensar la ciudad desde la perspectiva de la revalorización de los cuidados y la consideración de que estas tareas aún recaen en mayor medida sobre las mujeres, señalada tanto en los Planes de Igualdad como en las entrevistas realizadas. De ahí la insistencia en un urbanismo que favorezca la conciliación y, sobre todo, la corresponsabilidad -aspecto esencial de un urbanismo con perspectiva de género-, con medidas que faciliten el cuidado a través de acciones concretas como los Bancos de Tiempo, la asignación de recursos para cubrir necesidades de cuidado, la firma de un Pacto Local por la Conciliación entre ayuntamiento, partidos políticos y asociaciones de empresarios/as; así como acciones de sensibilización y formación para valorar los cuidados y la conciliación responsable o la ampliación y flexibilidad 
de los horarios de los servicios. Frente a un urbanismo con perspectiva de género a la manera tradicional (centrado en cuestiones de visibilidad o de seguridad) aquí se apuesta por un cambio más profundo en los valores que generan desigualdad y en medidas que hagan más porosas las fronteras entre lo público y lo privado, puesto que ahí radica una de las principales causas de la desigualdad entre hombres y mujeres.

Por todo esto, y a pesar de las carencias encontradas en los Planes o desveladas por las entrevistas, sí parece existir una conciencia de que el urbanismo no es neutro en lo que se refiere al género, tanto en Pamplona como en Tudela. De ahí que ambos municipios se planteen la necesidad de adoptar esta perspectiva de forma transversal en todas las áreas, y se apunten ya medidas concretas en esta línea ${ }^{11}$.

Más aún, pese a estas “carencias” conviene señalar también los límites del análisis de los Planes (de Urbanismo e Igualdad) de cara a la identificación de los intereses y sensibilidades -en esta u otras materias- de los respectivos equipos de gobierno municipales. Los planes pueden recoger las recomendaciones de los organismos internacionales, pero de nada valdrá este esfuerzo si no se refuerzan dichos documentos con los recursos (materiales, técnicos y humanos) necesarios para su implementación; o si ésta no se garantiza a través de la puesta en marcha de mecanismos de seguimiento, control y evaluación.

Así, por ejemplo, en Tudela, un municipio con muchos menos recursos y personal técnico en las áreas de Urbanismo e Igualdad (que ni siquiera se constituye como un área en sí misma), el Primer Plan de Igualdad (2006) recogía, casi punto por punto, todas las cuestiones e indicadores que aquí hemos manejado. Sin embargo, apenas se implementó; en 2013 se adoptó el II Plan, de corta duración (2013-2014) y que incorporaba la evaluación del anterior, reconociendo que apenas se había aplicado por un "exceso de ambición". El III y vigente plan de Tudela, pese a ser más modesto en sus objetivos, sí está dotado económicamente "de presupuesto real" (PTIT). De hecho, buena parte del éxito de las políticas - en este caso, inclusivas y sensibles a la perspectiva de género- tiene que ver con la implicación y sensibilización en materia de igualdad de género del personal al servicio de las administraciones públicas. Como ocurre en Tudela, la dedicación y entrega de miembros de la plantilla del Consistorio

11. En este sentido queremos señalar que la propia realización de las entrevistas ha servido para avivar la toma de conciencia por parte de los actores implicados en la elaboración de las políticas de la necesidad de unir los dos ámbitos analizados aquí: Urbanismo e Igualdad. Transmitir las recomendaciones del Programa Habitat sobre esta cuestión ya ha tenido, en sí, un efecto en este sentido. Pero el principal resultado en esta línea se ha derivado de la realización de la Mesa de Trabajo con representantes de Igualdad y Urbanismo de ambos Ayuntamientos para exponer los resultados de la investigación. Ha sido éste un espacio de encuentro en el que no solo se ha tomado mayor conciencia de la necesidad de colaboración, sino que se ha sembrado/reforzado una línea de actuación conjunta entre las dos áreas y también en colaboración con las Universidades, que pueda contribuir a unir espacios -académico y político-, tal como pretende hacerlo en la ciudad un urbanismo con perspectiva de género. 
a la causa de la igualdad y la no discriminación son una condición sine qua non para el triunfo de cualquier plan. Algo semejante podemos encontrar en el caso de Pamplona. El Área de Igualdad cuenta con presupuesto propio, pero las actuaciones del Plan competen a todas las áreas municipales, de ahí que resulte complicado valorar si se dota al Plan de recursos económicos suficientes. En cualquier caso, cada nuevo plan recoge las evaluaciones de los anteriores, destacando cómo la mayor parte de los objetivos alcanzados corresponden, en su mayor parte, al Área de Igualdad, incluyendo un volumen extraordinario de actividades por parte de esta área municipal, mientras que las acciones encaminadas a lograr la implicación de otras concejalías lograron un menor nivel de realización.

En cualquier caso, más allá de los planes y documentos concretos, la preocupación por determinados temas -como la transversalidad en los temas de género o la conciliación- puede evidenciarse en otros ámbitos y con otras medidas, que, en parte, compensan la falta de coordinación entre las áreas de Igualdad y Urbanismo en ambas ciudades: así, desde el Pacto por la Conciliación en Tudela, Igualdad ha trabajado por alianzas (puntuales y personales) con las áreas de deporte, educación, empleo y transporte ( $y$ menos con urbanismo y cultura). En el municipio se concede una gran importancia a estos Pactos Locales por la Conciliación de la vida laboral, familiar y personal, en los que la ciudadanía ha tenido un papel muy importante ${ }^{12}$. Asimismo, otros elementos atestiguan la importancia del género en la gestión municipal en Tudela: el Consejo Municipal por la Igualdad (que ha sustituido al Consejo de la Mujer, activo desde 1984), tiene amplia capacidad de intervención e incidencia en cuestiones de género. La histórica falta de personal en Igualdad se vio parcialmente corregida con la creación de la plaza de Técnica de Igualdad en 2007. La realización del "Estudio sobre la situación de las mujeres de Tudela" (2004), el de "La Historia de las mujeres de Tudela durante los últimos 60 años" (2010) o la creación de la Escuela de Empoderamiento, cuya consolidación es una de las estrategias a seguir en el IIIPIT, son otros indicadores.

Igualmente, también en Pamplona podemos encontrar acciones significativas encaminadas a favorecer la igualdad y la conciliación, como pueden ser los Talleres gratuitos y el Servicio de Atención Infantil en vacaciones escolares, organizados desde hace años por el Ayuntamiento y cuya oferta se ha ido ampliando progresivamente. Otro ejemplo es el Centro Ematic (anteriormente Centro Compañía), del Ayuntamiento de Pamplona, que desde 2002 ofrece información sobre igualdad de género, recursos de apoyo a la conciliación o para combatir la violencia contra las mujeres, o los Talleres de intercambio de tareas domésticas, que pretenden avanzar

12. Así, desde la firma del II Pacto, el Área de Transporte incluye la perspectiva de género. 
en la autonomía personal y la corresponsabilidad familiar, recordando que todas las tareas de la vida cotidiana son responsabilidad de hombres y mujeres por igual. La implicación en este tipo de acciones da muestra del compromiso real de los consistorios por contribuir al diseño de ciudades que sean sensibles e inclusivas por lo que al género se refiere; ciudades que favorezcan, no tanto la conciliación sino, más bien, la corresponsabilidad, que exige que tanto varones como mujeres puedan transitar fácilmente de un espacio a otro.

\section{Conclusiones}

A modo de conclusión podríamos señalar, en primer lugar, que tanto las entrevistas como los documentos analizados en ambos municipios recogen, no solo los temas clásicos de la seguridad y la visibilidad -a los que, efectivamente, los dos prestan una gran atención-, sino también los aspectos más novedosos del urbanismo con perspectiva de género, concretamente, la valoración de los cuidados en el diseño de la ciudad para facilitar que las mujeres puedan acceder a la ciudad en condiciones de igualdad. Esto implica apostar por una ciudad que favorezca la conciliación y, sobre todo, la corresponsabilidad. Este parece ser el núcleo de un urbanismo con perspectiva de género y su principal contribución a romper con las desigualdades que afectan a las mujeres.

Por ello, y a pesar de las carencias encontradas en los Planes o desveladas por las entrevistas, tanto en Pamplona como en Tudela, sí parece existir una conciencia de que el urbanismo no es neutro en lo que se refiere al género. De ahí que ambos municipios se planteen la necesidad de adoptar esta perspectiva de forma transversal en todas las áreas, y se apunten ya medidas concretas en esta línea.

Avanzar en esta dirección sería, por tanto, la principal recomendación que podría realizarse a ambos consistorios. Además, cabría un margen de mejora en la dimensión de la gobernanza, principalmente, en lo relativo a la recogida de datos/ indicadores desglosados por género. Esto proporcionaría una información de indudable valor, no solo para el diseño de nuevas políticas, sino, sobre todo, y más allá del compromiso a nivel propositivo, para medir, de alguna manera, el alcance/impacto real de las políticas de urbanismo en el ámbito de la igualdad de género.

Más aún, disponer de este tipo de datos desglosados permitiría, además, profundizar en la investigación en esta línea. Y, probablemente, ayudaría a conocer cuáles son las razones que podrían explicar el diferente grado de compromiso con un urbanismo con perspectiva de género. 


\section{Bibliografía}

ACERO, Guillermo, Jon AGUIRRE SUCH, Jorge AREVALO MARTIN, Pilar DIAZ RODRIGUEZ, Iñaki, ROMERO FERNANDEZ DE LARREA y Pedro HERNANDEZ MARTINEZ (2019) Planificación urbana, aprendiendo de Europa, Diputación de Barcelona: Barcelona.

BOCCIA, Teresa (2013) "Lempowerment economico delle donne nelle città, TRIA Territorio della Ricerca su Insediamenti e Ambiente, 10 (1), 23-34. https://doi. org/10.6092/2281-4574/1726

BOCCIA, Teresa (2020) "Ciudades sostenibles y género: el compromiso de las redes de mujeres para la Nueva Agenda Urbana", Ciudad y Territorio. Estudios Territoriales (CyTET), 52(203), 13-23.

CARTA EUROPEA DE LA MUJER EN LA CIUDAD (1995) Disponible en: http:// habitat.aq.upm.es/dubai/96/bp018.html.

DURÁN, María Ángeles (2010) Tiempo de vida y tiempo de trabajo, Madrid: Fundación BBVA.

DURÁN, María Ángeles (2018) La riqueza invisible del cuidado, Valencia: Universitat de València.

FALÚ, Ana (2013) "Cuestiones espaciales: la forma urbana de la ciudad influye en las posibilidades de la economía en particular para las mujeres", TRIA Territorio della Ricerca su Insediamenti e Ambiente, 6 (10), 35-42. https://doi. org/10.6092/2281-4574/1725.

I PLAN DE IGUALDAD DE OPORTUNIDADES ENTRE MUJERES Y HOMBRES DEL AYUNTAMIENTO DE TUDELA (2006). Disponible en http://www.tudela. es/docs/bienestar-social/plan-igualdad-tudela.pdf.

II PLAN DE IGUALDAD DE OPORTUNIDADES ENTRE MUJERES Y HOMBRES DEL AYUNTAMIENTO DE TUDELA 2013 - 2014. Disponible en http://www. tudela.es/docs/bienestar-social/2-plan-igualdad-tudela-2013-2014.pdf

III PLAN PARA LA IGUALDAD DEL AYUNTAMIENTO DE TUDELA 2018 - 2021. Disponible en http://www.tudela.es/uploads/archivos/iii-plan-deigualdad-30-01-2018.pdf.

III PLANPARALAIGUALDADDEMUJERESY HOMBRESDEL AYUNTAMIENTO DE PAMPLONA 2016 - 2022. Disponible en https://www.pamplona.es/temas/ igualdad-y-lgtbi/3-plan-igualdad.

INNERARITY, Carmen y SANCHO, Ana (2015) “Ciudad y ciudadanía. Un análisis de los planes estratégicos de desarrollo urbano desde la perspectiva de género" en Investigaciones Feministas, 5, 342-370. https://doi.org/10.5209/rev_INFE.2014. v5.48139 
MANSBRIDGE, Jane y OKIN, Susan Moller (2007) "Feminism”, en R. E. GOODIN; P. PETTIT, y T. POGGE (eds.) A Companion to Contemporary Political Philosophy. Volume I, Oxford: Blackwell, 332-359

MUXÍ, Zaida et al. (2011). “QQué aporta la perspectiva de género al urbanismo?”. Feminismo/s, 17, 105-129. http://dx.doi.org/10.14198/fem.2011.17.06

OKIN, Susan M. (1989) Justice, Gender and Family, Nueva York: Basic Books.

OKIN, Susan M. (1994) "Political Liberalism, Justice, and Gender”, Ethics, 105 (1), 23-43.

OKIN, Susan M. (2004) “The Public/Private Dichotomy”, en Colin FARRELLY (ed.). Contemporary Political Theory, Londres: Sage, 185-194.

ONU (1996a) Programa Habitat II. Disponible en: http://habitat.aq.upm.es/aghab/ aproghab.html

ONU (1996b) Declaración de Estambul sobre los Asentamientos Humanos. Disponible en: http://habitat.aq.upm.es/aghab/adeclestambul.html.

ONU (2017) Nueva Agenda Urbana (NAU). Secretaría de Habitat III. Disponible en: http://habitat3.org/wp-content/uploads/NUA-Spanish.pdf.

ORDENANZA PARA LA IGUALDAD ENTRE HOMBRES Y MUJERES DEL AYUNTAMIENTO DE PAMPLONA (OI 2019). Disponible en: https://www. pamplona.es/media/2511.

PATEMAN, Carol (1996) “Críticas feministas a la dicotomía público/privado” en C. CASTELLS (comp.) Perspectivas feministas en teoría política, Barcelona: Paidós, 31-53.

SÁNCHEZ DE MADARIAGA, Inés (2004) Urbanismo con perspectiva de género, Sevilla: Instituto Andaluz de la Mujer.

SÁNCHEZ DE MADARIAGA, Inés (2009) "Vivienda, movilidad y urbanismo para la igualdad en la diversidad: ciudades, género y dependencia”, Ciudad y Territorio. Estudios Territoriales (CyTET), (161-162), 581-598.

SANCHO, Ana (2016) "Habitat III: hacia una planificación sensible al género" TRIA, 16, 91-104. https://doi.org/10.6092/2281-4574/3970

SANCHO, Ana (2020) Ciudades conciliadoras. Urbanismo y género, Pamplona: Aranzadi.

YOUNG, Iris Marion (1996) "Vida política y diferencia de grupo: una crítica del ideal de ciudadanía universal”, en Carme CASTELLS (comp.) Perspectivas feministas en teoría política, Barcelona: Paidós, 99-126. 
